\title{
A strategy of traditional Chinese medicine against COVID-19: linking current basic research and ancient medicine texts
}

\begin{abstract}
A strategy of integrating Traditional Chinese Medicine (TCM) with Western medicine is proposed for better case management of the coronavirus disease (COVID-19) that is rapidly spreading in the world. We have reviewed the abundant classic TCM literature and from it linked several key texts to findings from biomedical research against SARS$\mathrm{CoV}-2$, including in silico studies of the efficacious compounds in TCM formulae. We have identified three TCM formulae: Yin Qiao San (銀超散), San Ju Yin (桑菊飲), and Xie Bai San ( 瀉白散) as they seem to hold high potentials against SARS-CoV-2 in COVID-19 patients. The strategy calls for early administration of these TCM formulae to decrease the rate of transformation from mild/moderate status into severe/critical status, thereby reducing the demand for ICU facility and ventilators.
\end{abstract}

Keywords: Coronavirus disease-19 (COVID-19), severe acute respiratory syndrome coronavirus 2 (SARS-CoV-2), traditional Chinese medicine (TCM), herb formula, antiviral, anti-inflammation
Volume 13 Issue 2 - 2020

\author{
Paul C Kwong,' Yuan-Chi Lin, ${ }^{2,3}$ Chung-Jen \\ Chen $^{4}$ \\ 'FormerWHO Consultant (TDR/Tropical Disease; GPA/Global \\ AIDS), Taiwan \\ ${ }^{2}$ Director, Medical Acupuncture Service \& Senior Associate in \\ Anesthesia and Pain Medicine, Children's Hospital Boston, USA \\ ${ }^{3}$ Associate Professor of Anaesthesia and Pediatrics, Harvard \\ Medical School, Boston, USA \\ ${ }^{4}$ Senior Attending Physician, Kaohsiung Municipal Ta-Tung \\ Hospital, Kaohsiung Medical University Hospital, Taiwan
}

\author{
Correspondence: Chung-Jen Chen, M.D., Dr. of TCM, \\ Kaohsiung Medical University,Taiwan 80I,Tel 886930590682 , \\ Email I088066@kmuh.org.tw
}

Abbreviations: COVID-19, Coronavirus disease-19; TCM, traditional Chinese medicine; SARS-CoV-2, severe acute respiratory syndrome coronavirus 2

\section{Introduction}

Coronavirus disease 2019 (COVID-19), caused by severe acute respiratory syndrome coronavirus 2 (SARS-CoV-2), is spreading rapidly in over 209 countries. As of 4 April 2020, over one million infected patients and over 60,000 deaths were reported by the WHO COVID-19 Situation Report-75. ${ }^{1}$ The case fatality rate of COVID-19 of $4.2 \%$ was 84 times higher than that of the flu pandemic. Although $80 \%$ of COVID-19 cases are mild or moderate, $20 \%$ of patients present with severe illness requiring further management such as hospitalization. A recent cohort study of 201 patients with confirmed COVID-19 pneumonia who were admitted and regarded as severe status to a medical center in Wuhan City, China showed that $41.8 \%$ of them developed acute respiratory distress syndrome (ARDS, regarded as critical status). Moreover, among those patients with ARDS, $52.4 \%$ died. $^{2}$ This implies that once developing ARDS, half of the patients could die. An effective anti-microbial therapy against SARS-CoV-2 is therefore needed. Although several chemical medications have been reported, ${ }^{3}$ there is no established therapy for SARS-CoV-2 at present.

\section{Mini review}

Traditional Chinese medicine (TCM) has a history of over $\quad 2000$ years. There are several ancient TCM texts that describe the application of TCM in severe flu-like disease, such as Shang Han Lun (傷寒論, Treatise on Febrile Diseases) in the Han Dynasty and Wen Bing Tiao Bian (溫病條辨, Detailed Analysis of Epidemic Warm Diseases) in the Ching Dynasty. These TCM texts may contain formulae which may have high potentials in treating COVID-19 cases. Another promising route is to use modern biomedical tools to identify and directly use the bioactive compounds in herbs. In 2003, facing the SARS outbreak, we attempted and succeeded to find an herb that might be effective against the coronavirus, SARS-CoV. An aqueous extract called TSL1 from the tender leaf of Toona sinensis Roem (香椿) was tested effective against SARS-CoV in vitro with a Selectivity Index of 12 to $17 .{ }^{4}$ The extract was subsequently tested with the pandemic influenza A (2009 H1N1) virus. Results showed that TSL-1 inhibited (SI > 15) viral yields on Madin-Darby Canine Kidney cell plaque formation by H1N1 virus on infected A549 cells. Further tests demonstrated that TSL-1 inhibited 2009 H1N1 viral activity by suppressing viral attachment onto the A549 cells. Moreover, compared to controls with the H1N1 drug amantadine, the TSL-1 treatment was associated with a better effect for down-regulation of adhesion molecules and chemokines (VCAM-1, ICAM-1, E-selectin, IL-8, and fractalkine), ${ }^{5}$ which are involved in pulmonary damage. After the Toona sinensis experiments, another team revealed that the herb Loranthi Ramulus ( 桑寄生) was even more effective against SARS-CoV in Vero E6 cells with SI $>92.8$, this time via anti-RNA replication pathways. ${ }^{6}$ The above findings suggest that TCM could be integrated with Western medicine against SARS-CoV-2 and the COVID-19 pandemic.

Zhang et al. ${ }^{7}$ from Shanghai, China, with population of over 20 million, has conducted an in silico screening of herbal medicines in TCM in order to find potent herbs against SARS-CoV-2. ${ }^{7}$ By crossreferencing screened herbs with three Chinese herbal databases and classic catalogue assignment and selection, they reported 26 Chinese herbs possessing very high potential against SARS-CoV-2, with nine classified as suitable for use during the full course of COVID-19.? As drug safety is the paramount consideration in clinical practice, we scrutinized the nine herbs and excluded three because of uncommon use in clinical practice or adverse effects. The result is that we take only six herbs for further consideration in clinical application. Based upon our strict criteria for drug safety, the final list includes: (1) Forsythiae fructus (連莬), (2) Licorice (甘草), (3) Mori cortex (桑白 皮), (4) Chrysanthemi flos (菊花), (5) Loricerae japonicae flos (金銀 花), and (6) Mori follum (桑葉). 
As is well known, the treatment strategy of dispensing herbs in TCM is to prescribe a TCM formula (方劑 known as Kampo 漢 方 in Japan) which consists of several herbs working together. It is uncommon to prescribe a single TCM herb. Hence, we took the list and consulted experts in TCM practice with the following question: which of the thousands of existing TCM formulae would be mostmatched to the six Chinese herbs that we have selected. This screening process narrowed the TCM selection to just three formulae, and they are known as (a) Yin Qiao San (銀㙓散, also Yin-Chiau San), ${ }^{8}$ (b) Sang Ju Yin (桑菊飲) ${ }^{9}$ and (c) Xie Bai San (瀉白散). ${ }^{10}$

The degree of "matching" of our herbs to the TCM formulae is shown in Table 1, Sang Ju Yin, having four herbs matched to the six key herbs on the list, a $67 \%$ of match rate, seems to have the highest potential against SARS-CoV-2. Next, Yin Qiao San with three herbs matched, has a $50 \%$ match rate. Finally, Xie Bai San with two herbs shows a $33.3 \%$ match rate. In the following section we report our review of how these three TCM formulae are described in TCM files or books. Our purpose is to clarify the clinical indication of the respective TCM formulae.

Table I Three most-matched TCM formulae matched with six herbs with high potential against SARS-CoV-2

\begin{tabular}{|c|c|c|c|}
\hline $\begin{array}{l}\text { TCM Formula } \\
\text { potential herbs } \\
\text { against SARS-CoV-2 }\end{array}$ & $\begin{array}{l}\text { Yin Qiao San } \\
\text { (A) }\end{array}$ & $\begin{array}{l}\text { Sang Ju Yin } \\
\text { (B) }\end{array}$ & $\begin{array}{l}\text { Xie Bai San } \\
\text { (C) }\end{array}$ \\
\hline Forsythiae fructus & + & + & - \\
\hline Licorice & + & + & + \\
\hline Mori cortex & - & - & + \\
\hline Chrysanthemi flos & - & + & - \\
\hline Loricerae japonicae flos & + & - & - \\
\hline Mori follum & - & + & - \\
\hline$\%$ of match* & 50 & 67 & 33 \\
\hline
\end{tabular}

*The number of herbal formulae (top rows) matched to any one of the key herbs (left column) divided by 6

We traced the origin of the formulae to an early source called Wen Bing Tiao Bian (溫病條辨), Detailed Analysis of Epidemic Warm Diseases). We found several impressive descriptions of the strategies taken by ancient TCM doctors, such as the following:

i. “All diseases of WEN (溫病, wen bing means some kind of infectious disease) originated from the upper body part, at the “Hand-Tai Yin" (which means the lung) [凡病溫者, 始於上焦, 在手太陰。]

ii. "Patients with Tai-Yin WEN disorder being afraid of WindCold, doctors should let them take the Gui Zhi Tang 桂枝湯 first; afterwards, if the patients' cold has been resolved but the other symptoms are still unresolved, then Yin Qiao San (銀趐散) would be the first choice to overcome them.

iii. If a patient displays symptoms of Tai-Yin Wind-WEN and keeps coughing and if the body is not very hot while feeling slightly thirsty, then prescribe the pungent-cool herbs. Sang Ju Yin is the first choice.

[太陰風溫, 但咳, 身不甚熱, 微渴者, 辛涼輕劑桑菊飲主 之。 Literal translation: Tai-Yin Wind-WEN, only cough, body being not very hot, light thirst, pungent and cool and drastic prescription, Sang Ju Yin is the first choice.]"

Based on the above description in classic text, we recommend that Sang Ju Yin be indicated for COVID-19 patients with cough in the early COVID-19 stage. Although $80 \%$ of COVID-19 patients are classified as mild or moderate, they can develop into severe cases if viral infections in their respiratory tracts get worse. This would usually be presented as dyspnea and could develop into ARDS. In this case, according to TCM clinical expert, Xie Bai San(瀉白散), invented by the TCM Dr. Qian Yi (錢乙) (1032-1113 AD) is strongly recommended. In summary, the TCM formulae shown in Table 2 are recommended for mild/moderate cases (not for severe status). The objective is to avoid patients progressing into severe cases ( $20 \%$ of all patients). As reported in hospital records, the mortality rate of of ARDS was 52.4\%. Among COVID-19 pneumonia cases, $41.8 \%$ developed ARDS; therefore, interrupting the progression of pneumonia into ARDS holds the key to decrease the fatal rate of COVID-19. ${ }^{2}$ From the above analysis, Sang Ju Yin and Xie Bai San could play a protective role prior to the development of ARDS. We suggest starting Sang Ju Yin plus Xie Bai San as soon as possible among COVID-19 pneumonia especially at the beginning of dyspnea and prior to ARDS.

Table 2 Recommended TCM formulae against COVID in cases with mild to moderate status (not for cases classified as severe status)

\begin{tabular}{ll}
\hline Status of COVID-I9 & Recommended TCM Formula \\
\hline $\begin{array}{ll}\text { Confirmed diagnosis (fever +/- sore } \\
\text { throat) }\end{array}$ & (A) Yin Qiao San \\
Confirmed diagnosis with Cough & (B) Sang Ju Yin \\
$\begin{array}{ll}\text { Confirmed diagnosis with Cough with } \\
\text { Dyspnea* }\end{array}$ & (B) Sang Ju Yin plus(C) Xie Bai San
\end{tabular}

*A close collaboration between TCM doctors and other medical team members to monitor and support the patients is important in particular at the onset of dyspnea. A combined therapy to integrate eastern and the western medication is advised rather than using either eastern or western medicine

The above three TCM formulae, Yin Qiao San, ${ }^{8}$ Sang Ju Yin ${ }^{9}$ as well as Xie Bai $\mathrm{San}^{10}$ consists of primarily herbs identified by Zhang et al. using in silico screening. ${ }^{7}$ In the course of pneumonia, overwhelming inflammation is another issue to be overcome. So, besides the antiviral therapy proposed by the above three TCM formulae, anti-inflammation therapy at the advance stage of pneumonia such as ARDS must be addressed. In western medicine, glucocorticoids therapy is a common option, but the adverse effect of it is that it could also prolong the life span of viruses. In TCM, instead, Ma Xing Gan Shi Tang, also known as Ma Xing Shi Gan Decoction (麻杏甘石湯 or 麻杏石甘湯) could be the first choice. Ma Xing Gan Shi Tang was found to have anti-inflammation effect and it could dampen the progression of pulmonary fibrosis, which is commonly seen in ARDS. ${ }^{11}$ Our view is thus: once cases of COVID-19 with pneumonia get worse (i.e. going to ARDS), Ma Xing Shi Gan Shi Tang could be administered.

We find a similar strategy from a medical authority on SARS and COVID-19 in China, Professor Boli Zhang (張伯禮) who propounded this important concept: "Use TCM to dampen the progression of COVID-19 from mild/moderate status to severe/critical status." According to his oral report in a TV interview about the recent battle 
of COVID-19 in Wuhan city, in a study of his cohort of 400 COVID-19 patients in Wuhan, there was "zero case that had progressed to severe/ critical status". By comparison, other teams in China and elsewhere relying solely on modern western medicine care (i.e. without TCM) were unable to match the same effect. ${ }^{2}$ Indeed, it is desirable to see a future study that compares TCM, western medicine and a combination of both strategies in treating COVID-19 cases during various stages of disease progression.

For hospitals, the question whether TCM can shorten the hospitalization days of COVID-19 patients remains to be answered.

Since there are critical shortages of ventilators and ICU facility in cities like New York and countries like Italy, we propose that the most prudent strategy is to adopt treatments that would effectively decrease the progress of COVID-19 into critical status. The currently emergent drug regime is hydroxychloroquine alone ${ }^{12}$ or in combination with azithromycin. ${ }^{13}$ However, the issue of their drug safety is widely concerned, especially in regard to the side effect of QT prolong among patients with cardiovascular disease comorbidity. At this juncture of the global COVID-19 pandemic, various strategies complement to hydroxychloroquine and azithromycin have been proposed.

Considering the very long history in clinical application for TCM formulae, we urge that TCM be considered as a complementary and integrative medical therapy for COVID-19, especially for cases contraindicated or intolerant to hydroxychloroquine or azithromycin. Furthermore, from the global public health point of view, our proposed strategy would contribute to the conservation of scarce intensive-care resources on the one hand, and, on the other, to the revival of traditional medicine in various cultures. The latter claim is demonstrated here by our effort to integrate several paradigms of therapy discovery, from database search for classic "indigenous" formulae to the employment of drug screening with in silico simulation of the ingredients of candidate formulae, and finally to actual clinical outcomes according to case records. We foresee a fertile ground in such a kind of vertical big-data TCM research that spans across disparate academic fields.

\section{Conclusion}

We recommend the followings:

a. TCM is an important resource and can be regarded as an option of complementary and integrative medical therapy against COVID-19.

b. Start using TCM formulae as early as possible for cases with confirmed diagnosis of COVID-19.

c. Administer TCM formulae (especially Yin Qiao San, Sang Ju Yin and Xie Bai San) in order to decrease the rate of transformation from mild/moderate to severe/critical status.

d. For cases entering severe/critical status, modern western medicine should be offered

e. In sum, incorporating TCM into the care of COVID-19 patients in mild to moderate disease status would likely reduce the burden on medical resources and avoid the collapse of modern medical care system during this urgent period of the COVID-19 pandemic.

\section{Acknowledgments}

The authors deeply thank the encouragement of Dr. Hseng-Kwang Hsu, Professor Emeritus, Department of Physiology, Kaohsiung Medical University, Kaohsiung, Taiwan. His dedicated help and support have been tremendously valuable throughout our investigation of coronavirus since 2003.

\section{Conflicts of interest}

Authors declare that there are no conflicts of interest.

\section{Funding}

None.

\section{References}

1. World Health Organization. COVID-19 situation report-75. Data as reported by national authorities by 12:00 CET 4 April 2020. Geneva, Switzerland, World Health Organization: 2020.

2. Wu C, Chen X, Cai Y, et al. Risk factors associated with acute respiratory distress syndrome and death in patients with Coronavirus disease 2019 pneumonia in Wuhan, China. JAMA Intern Med. 2020.

3. Martinez MA. Compounds with therapeutic potential against novel respiratory 2019 coronavirus. Antimicrob Agents Chemother. 2020. pii: AAC.00399-20.

4. Chen CJ, Michaelis M, Hsu HK, et al. Toona sinensis Roem tender leaf extract inhibits SARS coronavirus replication. J Ethnopharmacol. 2008;120(1):108-111.

5. You HL, Chen CJ, Eng HL, et al. The Effectiveness and Mechanism of Toona sinensis Extract Inhibit Attachment of Pandemic Influenza A (H1N1) Virus. Evid Based Complement Alternat Med. 2013:479718.

6. Wen CC, Shyur LF, Jan JT, et al. Traditional Chinese medicine herbal extracts of Cibotium barometz, Gentiana scabra, Dioscorea batatas, Cassia tora, and Taxillus chinensis inhibit SARS-CoV replication. $J$ Tradit Complement Med. 2011;1:41-50.

7. Zhang DH, Wu KL, Zhang X, et al. In silico screening of Chinese herbal medicines with the potential to directly inhibit 2019 novel coronavirus. $J$ Integr Med. 2020. pii: S2095-4964(20)30015-30017.

8. Lin SK, Tsai YT, Lo PC and Lai JN. Traditional Chinese medicine therapy decreases the pneumonia risk in patients with dementia. Medicine (Baltimore). 2016:95:e4917.

9. Zhang Y, Lyu C, Fong SYK, et al. Evaluation of potential herb-drug interactions between oseltamivir and commonly used anti-influenza Chinese medicinal herbs. J Ethnopharmacol. 2019;243:112097.

10. Lee KH, Yeh MH, Kao ST, et al. Xia-bai-san inhibits lipopolysaccharideinduced activation of intercellular adhesion molecule-1 and nuclear factorkappa B in human lung cells. J Ethnopharmacol. 2009;124(3):530-538.

11. Fei YX, Zhao B, Yin QY, et al. Ma Xing Shi Gan Decoction attenuates PM2.5 Induced lung Injury via Inhibiting HMGB1/TLR4/NFאB signal pathway in rat. Fron Pharmacol. 2019;10:1361.

12. Colson P, Rolain JM, Lagier JC, et al. Chloroquine and hydroxychloroquine as available weapons to fight COVID-19. Int $J$ Antimicrob Agents. $2020 ; 105932$

13. Gautret P, Lagier JC, Parola P, et al. Hydroxychloroquine and azithromycin as a treatment of COVID-19: results of an open-label non-randomized clinical trial. Int J Antimicrob Agents. 2020:105949. 